This item was submitted to Loughborough's Research Repository by the author.

Items in Figshare are protected by copyright, with all rights reserved, unless otherwise indicated.

\title{
A framework methodology for the simulation and sizing of diaphragm filter presses
}

PLEASE CITE THE PUBLISHED VERSION

PUBLISHER

(C) Elsevier

VERSION

AM (Accepted Manuscript)

LICENCE

CC BY-NC-ND 4.0

\section{REPOSITORY RECORD}

Wakeman, Richard J., and E.S. Tarleton. 2009. "A Framework Methodology for the Simulation and Sizing of Diaphragm Filter Presses”. figshare. https://hdl.handle.net/2134/5631. 
This item was submitted to Loughborough's Institutional Repository (https://dspace.lboro.ac.uk/) by the author and is made available under the following Creative Commons Licence conditions.

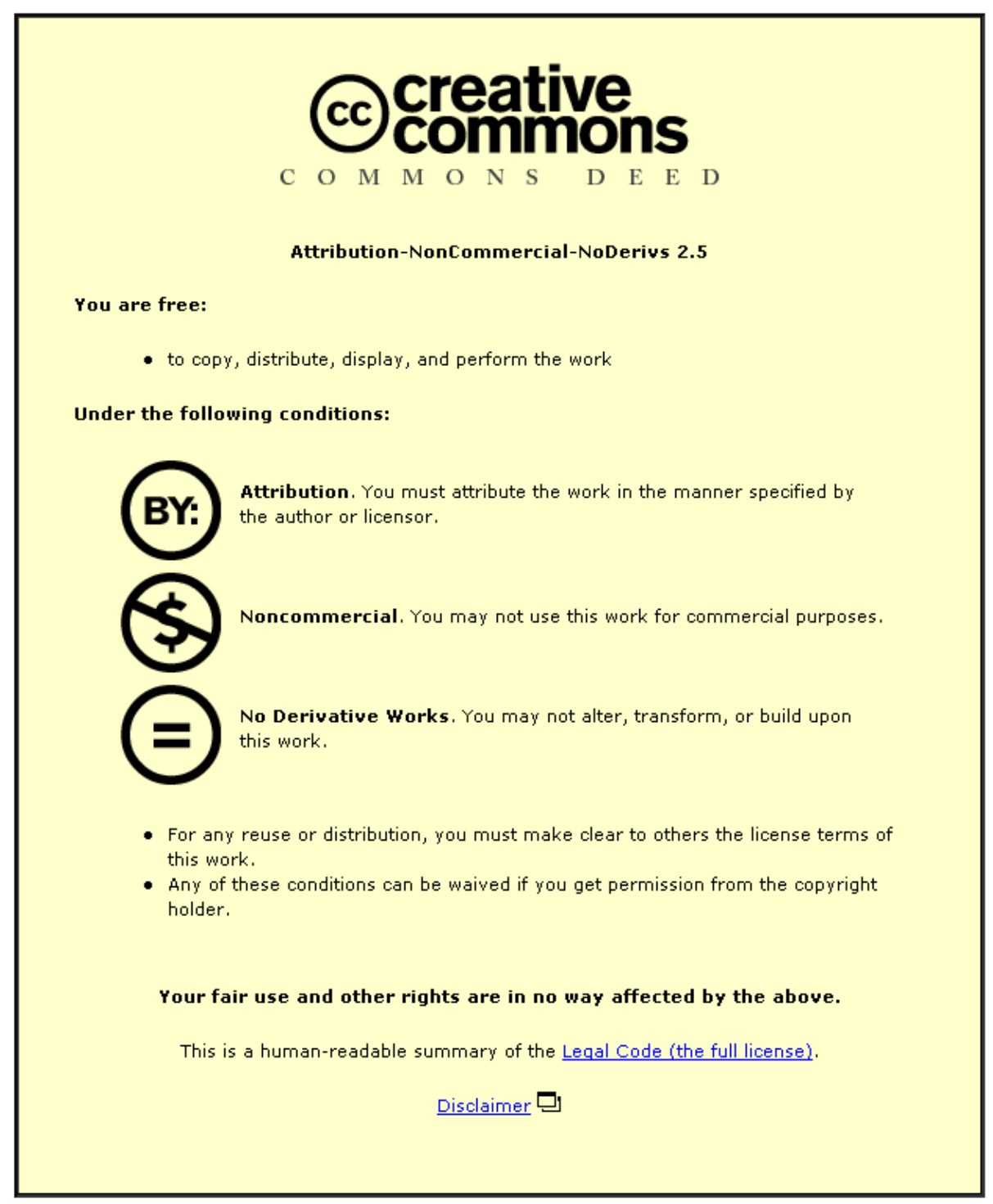

For the full text of this licence, please go to: http://creativecommons.org/licenses/by-nc-nd/2.5/ 


\title{
A FRAMEWORK METHODOLOGY FOR THE SIMULATION AND SIZING OF DIAPHRAGM FILTER PRESSES
}

\author{
R.J. Wakeman ${ }^{\dagger}$ and E.S. Tarleton (e.s.tarleton@lboro.ac.uk) ${ }^{\ddagger}$ \\ ${ }^{\dagger}$ Separation Processes Centre, University of Exeter, Devon, UK. \\ ${ }^{\ddagger}$ Dept. of Chemical Engineering, Loughborough University, Loughborough, Leics., UK.
}

\begin{abstract}
A framework for simulating filter cycles is presented, based on a blend of tested theoretical models and accepted design procedures. Output from the simulator predicts correctly the general effects of process variables. Some sample results are used to illustrate these when it is applied in a general sense to a diaphragm (variable volume) filter press.
\end{abstract}

\section{INTRODUCTION}

Of the many unit operations available to the process engineer, those associated with solid/liquid separation are perhaps the most difficult to specify. For these systems it would be rare practice to use fundamental theoretical relationships for either the selection, design or optimization of equipment with the result that the specification of chemical plant is rarely performed without recourse to extensive laboratory and pilot scale tests. Whilst the task of electing a solid/liquid separator for a given duty has been considerably simplified by the recent introduction of the computer software $\mathrm{p}^{\mathrm{C}}$-SELECT ${ }^{1,2}$, software for filter design is generally not available. When a particular type of separator has been identified, the separations technologist is still faced with the prospect of undertaking an extensive test programme to enable scale-up and optimisation of separator size and configuration. These problems are compounded by the dearth of literature relating to such matters and the apparent lack of 'standard' procedures.

Solid/liquid separation technology, whether it be in the areas of selection, design or optimisation, is best dealt with by interactive computer software. To be most effective the software must incorporate a well chosen mix of algorithms, expert system and input information from the user, be based on sound ideas and proven data from experimental and theoretical research projects, and maintain the ability to give advice and education in a congenial fashion so that any psychological barriers to computer use are avoided.

This paper describes some preliminary results from a software package designed to simulate the operation of pressure filters, using data based on the work reported previously ${ }^{3-7}$, with the aim of demonstrating the general usefulness of the approaches taken in that work. The software also lays the foundation for design procedures which may incorporate more complex models for cake formation ${ }^{8-10}$, which are based on more rigorous models of suspension flows and particle deposition. The software utilises industrially proven methodologies which are combined with computer code to produce modular routines capable of modelling cake formation, compression dewatering and washing. The techniques used to describe the operation of the diaphragm filter press are shown and information regarding a typical filter cycle is presented.

\section{FILTER CYCLE DESCRIPTION}


The versatility of the diaphragm filter press allows the range of operating configurations shown in Figure 1. Slurry is fed into the chambers of the press through suitable porting with either a centrifugal, diaphragm, single screw or reciprocating pump ${ }^{11}$ such that filtration can occur at constant rate/variable pressure or variable rate/variable pressure, depending on the type of pump used. Cake is formed simultaneously on the two opposing sides of the filter chamber, with the cake surfaces growing towards each other. If the chambers are not completely filled by cake during this primary filtration stage then a secondary filtration stage may follow where the suspension remaining between the cakes is filtered at constant pressure by movement of the flexible diaphragm located on one filtering surface. During this stage (ii) process, filtrate is collected from only one drainage surface whilst further cake also forms on the same surface. When sufficient movement of the diaphragm has occurred and suspension has been filtered for the surfaces of the two cakes to meet as a result of the primary and/or secondary filtration stages, further cake consolidation by the diaphragm (at constant pressure), cake dewatering by air blowing, and/or cake washing can be undertaken to complete the filter cycle prior to cake discharge. In any single filter cycle, a selection of the stages shown on Figure 1 may be utilised; some stages may be repeated within a cycle, and the order of utilisation can differ between applications. Any simulation methodology must be flexible enough to allow any combination of stages (i) to (v).

\section{Stages (i) and (ii): Cake Formation}

It is most common to describe filtration processes by the general filtration equation for the reciprocal volume flow rate of filtrate:

$$
\frac{d t_{f}}{d V_{f}}=\frac{\alpha_{a v} c_{c} \mu}{A^{2} \Delta p} V_{f}+\frac{\mu R_{m}}{A \Delta p}
$$

where $\alpha_{a v}$ is the specific resistance of the cake averaged over pressure to the applied pressure difference $\Delta p, \mu$ is the filtrate viscosity, $A$ is the filter area, $R_{m}$ is the resistance of the filter medium, and the effective concentration of solids in the feed, $c_{c}$, is given by

$$
c_{c}=\frac{\rho M_{s}}{1-m M_{s}}
$$

$\rho$ is the density of the filtrate, $M_{s}$ is the mass fraction of solids in the feed suspension, and $m$ is the ratio of the mass of the wet cake to the mass of the dry cake. In the most general sense, both the pressure and rate can vary and it is necessary to impose the pump characteristics on the governing equations. Hence equation (1) can be arranged to:

$$
V_{f}=\frac{1}{c_{c} \alpha_{a v}}\left(\frac{A^{2} \Delta p}{\mu Q}-A R_{m}\right)
$$

where $\alpha_{a v}$ is a function of the pressure applied to the solid/liquid mixture by the pump. To account for the potential compressibility of the cake, it is common practice to relate the specific resistance $(\alpha)$ and porosity $(\varepsilon)$ or voids ratio $(e)$ of the filter cake (evaluated at a particular pressure) to the solids compressive pressure through so-called constitutive equations ${ }^{12,13}$ similar to:

$$
\alpha=\alpha_{0} p_{s}^{n} \quad \text { or } \quad \alpha=\alpha_{0}\left(1+p_{s}\right)^{n}
$$




$$
\begin{aligned}
& \varepsilon=\varepsilon_{0} p_{s}^{a} \quad \text { or } \quad \varepsilon=\varepsilon_{0}\left(1+p_{s}\right)^{a} \\
& e=e_{0}-b \log \left(1+p_{s}\right)
\end{aligned}
$$

Although recent work ${ }^{5-7}$ has found equation (6) to be a better representation of available data than one of the forms of equation (5), the latter will be used here for illustrative purposes. Combining the integrated form of equation (4) (to give an average cake resistance) with equation (3) and performing a mass balance on the solids in the cake yields the two equations governing cake formation during the primary filtration phase (Stage (i)). The filtrate volume produced is

$$
V_{f}=\frac{1}{c_{c} \alpha_{0}(1-n) \Delta p_{c}^{n}}\left(\frac{A^{2} \Delta p}{\mu Q}-A R_{m}\right)
$$

and the total thickness of cake in the chamber is

$$
h=2\left(\frac{V_{f} M_{s}\left(\frac{\rho_{s}}{\rho}(m-1)+1\right)}{A \frac{\rho_{s}}{\rho}\left(1-m M_{s}\right)}\right)
$$

When unfiltered suspension remains in the filter chamber(s) a secondary filtration phase may follow the initial primary filtration. If the secondary phase occurs, then further filtration takes place at constant pressure (with the pressure applied by the diaphragm rather than the pump), with filtrate draining through one filtration surface in each chamber. Modifying equation (1), to account for the effective increase in resistance to filtration due to the presence of a filter cake on the filter medium at the start of the filtration, gives the governing equation:

$$
\frac{t_{f}-t_{t r}}{V_{f}-V_{t r}}=\frac{\alpha_{a v} c_{c} \mu}{(A / 2)^{2} \Delta p}\left(V_{f}+V_{t r}\right)+\frac{\mu}{(A / 2) \Delta p}\left(\frac{\alpha_{a v} c_{p} V_{p}}{(A / 2)}+R_{m}\right)
$$

Thus, if the pump characteristics relating $\Delta p$ and $Q$, and the constitutive equations relating $\alpha$ and $\varepsilon$ (and hence $m$ ) to $\Delta p$, are known from laboratory scale tests, cake formation rates can be calculated.

\section{Stage (iii): Cake Consolidation}

Cake consolidation is performed at constant pressure through use of a filter diaphragm and is analysed via an empirical relationship relating the consolidation ratio

$$
U_{c}=\frac{h_{o}-h}{h_{o}-h_{\infty}}
$$

to a dimensionless consolidation time defined by

$$
T_{c}=\frac{n_{1}^{2} C_{c} t_{c}}{\omega_{0}^{2}}
$$


such that

$U_{c}=\frac{\sqrt{4 T_{c} / \pi}}{\left(1+\left(4 T_{c} / \pi\right)^{v}\right)^{1 / 2 v}}$

where $C_{c}$ is the consolidation coefficient and $v$ the consolidation behaviour index. Values for $v$ and the other scale-up constants in the constitutive equations for the consolidation phase can be obtained through laboratory tests with, for instance, a leaf filter or a piston press (as previously described ${ }^{3-7}$ and related through equations (10)-(12) to give expressions for cake height and filtrate volume during the consolidation period(s).

Consolidation is assumed to end when the flow rate of liquid from the filter falls below a predetermined, usually uneconomic, level.

\section{(iv) Cake Dewatering: (by Air Blowing)}

The dewatering phase is analysed through a combination of empirical and theoretical relations. These facilitate the calculation of the time required to achieve a given filter cake moisture content and the air flow rate through the cake during the dewatering period, and have been described in detail ${ }^{14-16}$. Two parameters of prime importance in dewatering are the pressure required to initiate dewatering $\left(p_{b}\right)$ and the irreducible saturation of the filter cake $\left(S_{\infty}\right)$. Although these should be measured experimentally, the former can be determined with sufficient accuracy from

$$
p_{b}=\frac{4.6\left(1-\varepsilon_{a v}\right) \sigma}{\varepsilon_{a v} x_{a v}}
$$

Correlations exist (for relatively large, granular materials) which allow estimation of $S_{\infty}$, for example

$$
\begin{aligned}
& N_{c a p}=\frac{\varepsilon_{a v}^{3} x_{a v}^{2}\left(\rho g h+\Delta p_{d}\right)}{\left(1-\varepsilon_{a v}\right)^{2} h \sigma} \\
& S_{\infty}=0.155\left(1+0.031 N_{c a p}^{-0.49}\right)
\end{aligned}
$$

Similar correlations are not available for fine particle cakes, and the above should not be used if alternative data are available.

With data for $p_{b}$ and $S_{\infty}$ available, and assuming that no dewatering of the cake occurs through evaporation of the mother liquor and that the displacing fluid is saturated instantly as it enters the cake, a dimensionless saturation $\left(S_{R}\right.$, termed the reduced saturation) can be related empirically to a dimensionless time $(\theta)$ by

$$
S_{R}=\frac{S-S_{\infty}}{1-S_{\infty}}
$$


$\theta=\left(\frac{1}{b_{2}}\left(\frac{1}{S_{R}}-1\right)\right)^{1 / b_{3}}$

where $b_{2}$ and $b_{3}$ are constants determined by fitting equation (18) to numerical solutions of the differential equations describing simultaneous gas and liquid flow through the filter cake ${ }^{14,15}$. The dewatering time $\left(t_{d}\right)$ is then estimated from

$t_{d}=\frac{\theta \varepsilon_{a v} \mu h^{2}\left(1-S_{\infty}\right)}{\Delta p_{d} k_{a v}}$

Allowing for losses in the feed pipes to the filter and the effects of plant elevation, the air flow rate required to achieve the desired moisture content can be calculated ${ }^{15-17}$, and the gas or air blower sized accordingly.

\section{(v) Cake Washing: (by Displacement)}

The technique used in the analysis of washing phase(s) employs the dispersion model ${ }^{17-21}$ to interpret the rates of solute mass transfer from the voids in the filter cake. Use of the model requires a superficial wash velocity, which is estimated from the modified Darcy equation

$u_{o}=\frac{\Delta p_{w}}{\mu_{w}\left(\alpha_{a v} \rho_{s} h\left(1-\varepsilon_{a v}\right)+R_{m}\right)}$

and the product of the Reynolds and Schmidt numbers, calculated from

$\operatorname{Re} . S c=\frac{u_{o} x_{a v}}{\varepsilon_{a v} D}$

The dispersion coefficient varies with the filter cake thickness ${ }^{20}$, and is calculated from

$$
\begin{aligned}
& \frac{D_{L}}{D}=0.707+55(\operatorname{Re} . \mathrm{Sc})^{0.96} \quad 0 \leq h \leq 0.1 \mathrm{~m} \\
& \frac{D_{L}}{D}=0.707+1.75(\operatorname{Re} . \mathrm{Sc}) \quad h>0.1 \mathrm{~m}
\end{aligned}
$$

The dispersion number is then given by

$$
D_{n}=\frac{(\operatorname{Re} . \mathrm{Sc}) h}{x_{a v} \frac{D_{L}}{D}}
$$

If the degree of washing is specified in terms of the required removal of solute, the number of wash ratios used in the washing can be calculated using design charts derived from solutions of the differential equations describing solute dispersion in filter cakes ${ }^{18-21}$. With a knowledge of the number of wash ratios to be applied the washing time $\left(t_{w}\right)$, effluent solute concentration $(c)$ and fractional 
solute recovery $(F)$ can be estimated. Allowances are made for cake saturation at the start of washing, and for the scale and type of filter being used for the separation ${ }^{17,18}$.

\section{AN EXAMPLE OF FILTER SIMULATION}

The above methodologies enable flexible simulations to be developed. When suitably applied these can model most combinations of cake formation, consolidation, dewatering and washing to allow preliminary sizing and scale-up of equipment to be performed. Although the example of a diaphragm press has been chosen, the procedures can be readily adapted to model the performance of other filtration devices such as tube presses and rotary vacuum filters. The data shown below illustrate how information associated with the entire filter cycle can be produced for a set of typical operating conditions through software simulation techniques.

\section{Example Problem}

A diaphragm filter press has been identified as suitable for producing a nominal 5 te of dry particulate solids from an $8 \% \mathrm{w} / \mathrm{w}$ slurry. Following the formation stage(s), the homogeneity of the cake is to be improved through consolidation with the diaphragm. The resultant compact is then to be dewatered by air blowing until a moisture content of $20 \%$ is achieved, and subsequently washed until the solute concentration in the cake is reduced from 30 to $2.5 \mathrm{~kg} \mathrm{~m}^{-3}$. The feed is delivered to the filter press via a centrifugal pump (see Table 1 for the characteristic curve of the pump), and the feed suspension characteristics have been determined experimentally.

Filter and septum characteristics:

Filter chamber thickness

Filter medium resistance

Operating conditions:

Secondary filtration pressure (if needed)

Consolidation and washing pressure

Dewatering pressure

Barometric pressure

Solids concentration in feed

Solute concentration in feed

Solute concentration in wash

Cake properties:

Max. cake thickness on each filter surface

Constitutive equations during filtration

Constitutive equations during consolidation

Consolidation behaviour index

Particle and fluid properties:

Density of solids

Mean size of solids in feed

Density of filtrate and wash

Viscosity of filtrate and wash

Surface tension of filtrate and wash
$80 \mathrm{~mm}$

$3 \times 10^{11} \mathrm{~m}^{-1}$

$600 \mathrm{kPa}$

$600 \mathrm{kPa}$

$400 \mathrm{kPa}$

$100 \mathrm{kPa}$

$8 \% \mathrm{w} / \mathrm{w}$

$30 \mathrm{~kg} \mathrm{~m}^{-3}$

$0.5 \mathrm{~kg} \mathrm{~m}^{-3}$

$20 \mathrm{~mm}$

$\alpha=6 \times 10^{9} \Delta p^{0.6}$

$e=3-0.1 \log \Delta p$

$C_{c}=1 \times 10^{-8} \Delta p^{0.1}$

$e=1.63-0.55 \log \Delta p^{0.1}$

3
$2500 \mathrm{~kg} \mathrm{~m}^{-3}$
$10 \mu \mathrm{m}$
$988 \mathrm{~kg} \mathrm{~m}^{-3}$
$0.001 \mathrm{~Pa} \mathrm{~s}$
$0.07 \mathrm{~N} \mathrm{~m}^{-1}$ 
Irreducible cake saturation

Viscosity of air

Solute diffusivity
0.26

$1.8 \times 10^{-5} \mathrm{~Pa} \mathrm{~s}$

$10^{9} \mathrm{~m}^{2} \mathrm{~s}^{-1}$

The problem is to determine if the separation can be achieved, the filter cycle time, the filter area, and other performance indicators.

\section{Solution via Simulation}

When the computer simulation package is used to model the above problem a wealth of data related to both the individual phases and the total filter cycle is produced. Figures 2 to 7 give examples, where the simulations for the above data are given by the open circles (o) on each plot. The data suggest that the required objectives of the separation can be readily achieved with the diaphragm press and pump specified. The filter area and total cycle time are estimated to be $380 \mathrm{~m}^{2}$ and $6311 \mathrm{~s}$, with the filtration, consolidation, dewatering and washing phases projected to end after $2028 \mathrm{~s}(0.56$ $\mathrm{h}), 4056 \mathrm{~s}(1.13 \mathrm{~h}), 4707 \mathrm{~s}(1.31 \mathrm{~h})$ and $6311 \mathrm{~s}(1.75 \mathrm{~h})$ respectively.

During the period of the primary filtration the cakes in each of the filter chambers progressively grow to a combined thickness (noting that cake formation occurs on two surfaces in each chamber) of $34 \mathrm{~mm}$ (Figure 4), whilst the pressure in the chambers increases from 49.7 to $94 \mathrm{kPa}$ (Figures 2 and 3) as the cakes form. At the end of the primary filtration period $\left(t_{f}=1140 \mathrm{~s}\right)$ unfiltered suspension remains in the chambers and a secondary filtration at constant pressure is required. Within this period the cakes in each chamber continue to form and eventually join to achieve the maximum allowable thickness of $40 \mathrm{~mm}$ (i.e. 2x20 mm) (Figure 4) after $2028 \mathrm{~s}$. At the end of the cake formation stages the chamber and cake moisture contents are identical and equal to $52.1 \%$ with the total solids, liquid and solute masses in the filter estimated at 5092, 5534 and $166 \mathrm{~kg}$ respectively. The filtrate flow rate decreases progressively as cake is formed (Figure 5 ), with a very rapid decline corresponding to the secondary filtration caused by the diaphragm.

Following the cake formation phases the consolidation, dewatering and washing stages are sequentially initiated. During the period of consolidation the cake moisture and solute mass are reduced to $27 \%$ and $57 \mathrm{~kg}$ respectively, whilst the cake thickness is reduced to $21 \mathrm{~mm}$ (Figure 4 ) and is assumed to remain at that value for the subsequent dewatering and washing phases. The solute mass and moisture content of the cake are further lowered to $38 \mathrm{~kg}$ and the desired $20 \%$ (Figure 7) respectively at the end of the dewatering period, using an overall air rate of $1.8 \times 10^{-5} \mathrm{~m}^{3} \mathrm{~m}^{-2} \mathrm{~s}^{-1}$. In the washing period, about two wash ratios (based on the unsaturated cake) are used to produce the required solute concentration of $2.5 \mathrm{~kg} \mathrm{~m}^{-3}$; the fractional recovery for the washing period alone is $88 \%$. This final wash increases the moisture content of the cake to over $27 \%$ (Figure 7 ), that is, to its value at the end of the consolidation (stage (iii)) in the cycle when the cake was last fully saturated. The filtrate and wash liquor rates for the whole cycle are shown on Figure 6 . The wash liquor rate shown on Figure 6 is greater than the filtrate rate at the end of consolidation (stage (iii)) due to the different methods of pressure application and transmission through the filter chamber. During consolidation, the $600 \mathrm{kPa}$ is applied by the diaphragm and the stress is transmitted mainly through the solid phase in the cake; as consolidation proceeds, an increasing proportion of the applied stress is transmitted in this way. During washing, the $600 \mathrm{kPa}$ is applied in the liquid phase, and the only stress transmitted to the solids is that which results from drag at the particle surfaces. Hence, during washing the flow of liquid is higher than it is at the end of consolidation (stage (iii)) but lower than at the end of filtration (stages (i) and (ii)).

At the end of the filter cycle it is estimated that $5092 \mathrm{~kg}$ of solids, $1891 \mathrm{~kg}$ of liquid, and $4.7 \mathrm{~kg}$ of solute remain in the filter press. 


\section{EFFECTS OF THICKER CAKE FORMATION}

It is well known in practice that greater productivity is obtained from filters if thinner cakes are formed, and if the simulator functions properly it should predict such behaviour. To do this the data shown earlier is used, but the maximum cake thickness at the end of either stage (i) or (ii) on each filter surface is changed to $30 \mathrm{~mm}$ and $40 \mathrm{~mm}$ (obviously a $40 \mathrm{~mm}$ thickness completely fills the chamber, hence no secondary filtration (stage (ii)) occurs in this instance). The calculated data are compared with the $20 \mathrm{~mm}$ cake case on Figures 2 to 7 . Some effects of the thickness of cake formed during stages (i) and (ii) are summarised on Table 2.

Effects of allowing further cake growth in the filter are not obvious a priori because of the interactions between so many of the variables. Cakes are formed at slower rates as they become thicker, dependent on the shape of the pump characteristic curve. The pressure difference across a thicker cake is greater. This could potentially cause more compaction of the cake if it is compressible, but the time taken to compact a thicker cake is considerably greater than that needed to compact a thinner one, and hence the filtrate flux from a thicker cake tends to be rather lower. An uneconomic flux is therefore reached when the cake is in a less consolidated state, leaving the 'thicker' cake with a more open structure. Hence, even though the mean specific resistance will tend to increase at higher pressures, whilst the voids ratio will decrease, the net effect of these changes will be to reduce filtrate rates (as indicated on Figure 5) during cake formation (stages (i) and (ii)). (In this example, during consolidation (stage (iii)) the thickness of the $40 \mathrm{~mm}$ cake (per filter surface) is reduced by $22 \%$ to 31 $\mathrm{mm}$ and that of the $20 \mathrm{~mm}$ cake by $47 \%$ to $10.5 \mathrm{~mm}$.) The more open structure of the final cake is reflected in the higher wash liquor rates (Figure 6) and higher final cake moisture contents (Figure 7) associated with the thicker cake.

Increasing the cake thickness causes a number of changes in the cycle which impact on the economics of the separation. The main factors to consider are:

i. the extension of the cycle time, with the greatest extension occurring during stage (iv) (cake dewatering) in the cycle simulated in this example

ii. higher energy consumption per unit mass of product can be associated with longer cycle times

iii. a reduction in the overall solids (or filtrate) productivity rate

iv. an increase in the wash ratio (or the total volume of wash liquor used) in stage (v) (this is particularly important if wash liquor is to be regenerated thermally for re-use, as would be the case when a solvent wash is used)

v. a reduction in the filtration area required for the separation.

Of these factors, (i) to (iv) tend to increase separator operating costs (or decrease revenues in the case of (iii)), whilst (v) reduces capital (and to a lesser extent maintenance) costs. Hence, for any particular cycle configuration there may exist an optimum economic cake thickness which should be formed during the filtration stages of the cycle. In practice, it may also be possible to investigate different cycle configurations to minimise the costs.

\section{CONCLUSIONS}


This paper outlines the broad methodology behind the simulation of filter cycles, using the diaphragm filter press as an example. It indicates how computer simulations can significantly improve the understanding of the operations which can occur during the filter cycle. Each of the theories used to model the cake formation, consolidation, dewatering and washing phases has been proven in pilot scale and industrial tests, but are capable of considerable improvement from a fundamental point of view. It is apparent that the modelling procedures adopted for the simulation of the diaphragm filter press can be readily adapted to include optimisation routines and extended to encompass other types of separation equipment. When fully developed, such computer software should provide a suite of programs which are sufficiently powerful to minimise the number of costly and time consuming experiments which need to be performed to assess the suitability of equipment for a particular duty.

\section{NOMENCLATURE}

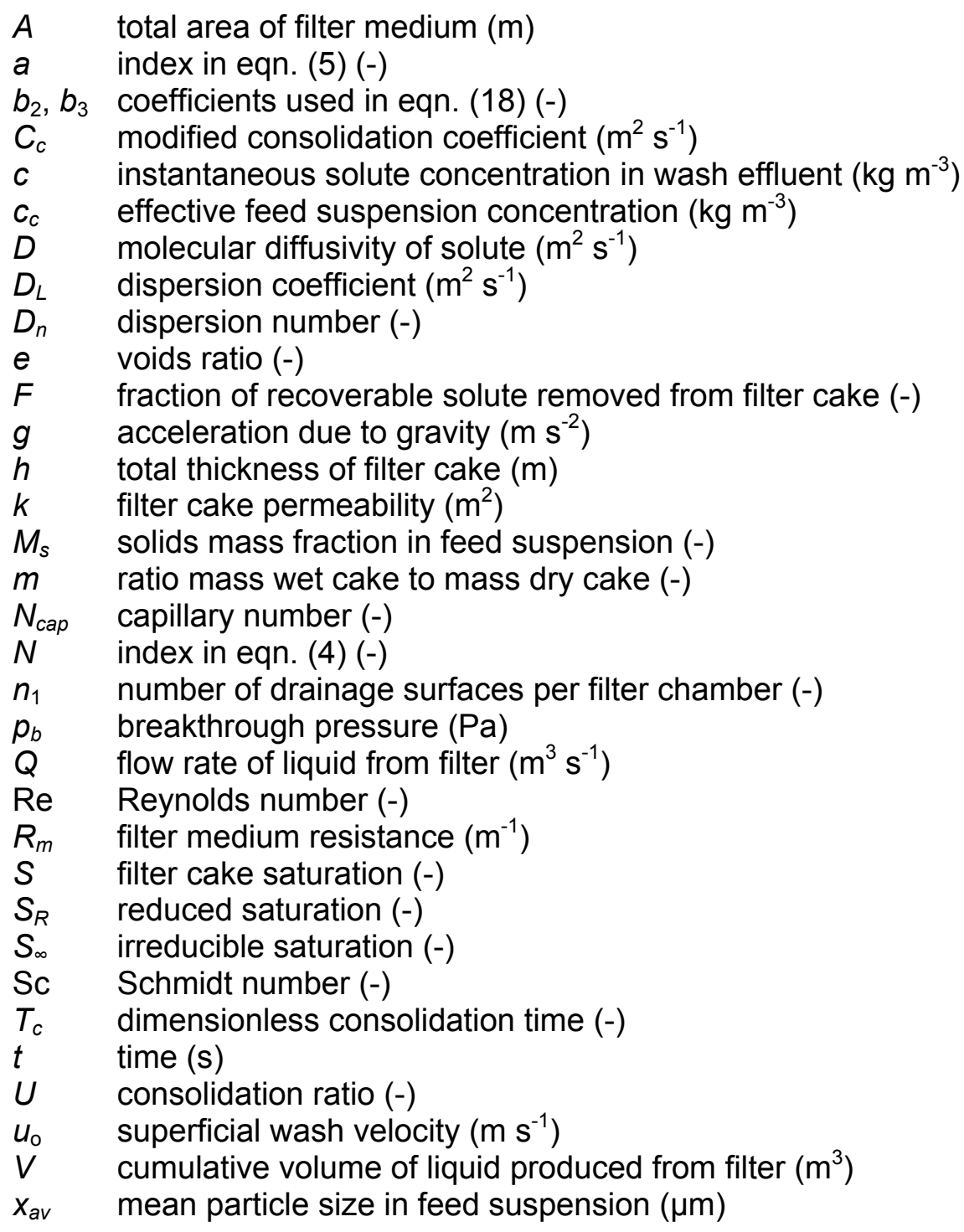

Greek symbols 
$\alpha \quad$ local specific cake resistance $\left(\mathrm{m} \mathrm{kg}^{-1}\right)$

$a_{a v} \quad$ average specific cake resistance $\left(\mathrm{m} \mathrm{kg}^{-1}\right)$

$\alpha_{0} \quad$ specific cake resistance at $\Delta p=1 \mathrm{kPa}\left(\mathrm{m} \mathrm{kg}^{-1}\right)$

$\Delta p \quad$ pressure applied to solid/liquid mixture ( $\mathrm{Pa}$ or $\mathrm{kPa})$

$\Delta p_{c} \quad$ pressure drop over filter cake $(\mathrm{Pa})$

$\varepsilon \quad$ local filter cake porosity (-)

$\varepsilon_{a v} \quad$ average porosity of filter cake (-)

$\varepsilon_{0} \quad$ filter cake porosity at $\Delta p=1 \mathrm{kPa}(-)$

$\theta \quad$ dimensionless time (-)

$\mu \quad$ liquid viscosity ( $\mathrm{Pa} \mathrm{s}$ )

$v \quad$ consolidation behaviour index (-)

$\rho \quad$ liquid density $\left(\mathrm{kg} \mathrm{m}^{-3}\right)$

$\rho_{s} \quad$ solids density $\left(\mathrm{kg} \mathrm{m}^{-3}\right)$

$\sigma \quad$ liquid surface tension $\left(\mathrm{N} \mathrm{m}^{-1}\right)$

$\omega_{0} \quad$ volume of solids produced per unit area of drainage surface $\left(\mathrm{m}^{3} \mathrm{~m}^{-2}\right)$

\section{Subscripts}

c consolidation phase

d dewatering phase

$f \quad$ filtration phase

o start of phase

$p \quad$ end of previous phase

tr transition from primary to secondary filtration phase

$w \quad$ washing phase

$\infty \quad$ at equilibrium

\section{REFERENCES}

1. Tarleton E.S. and Wakeman R.J., Solid/Liquid Separation Equipment Simulation and Design: $p^{C}$ SELECT - Personal Computer Software for the Analysis of Filtration and Sedimentation Test Data and the Selection of Solid/Liquid Separation Equipment, Separations Technology Associates, (1991).

2. Wakeman R.J. and Tarleton E.S., Solid/liquid separation equipment simulation and design - an expert systems approach, Filtration and Separation, 28(4), 268-274 (1991).

3. Wakeman R.J., Sabri M.N. and Tarleton E.S., Factors affecting the formation and properties of wet compacts, Powder Technology, 65(1-3), 283-292 (1991).

4. Wakeman R.J., Scale-up procedures and test methods in solid/liquid separation: 1. Test methods and calculation procedures for pressure filtration, Proc. $13^{\text {th }}$ Annual Meeting of International Fine Particle Research Institute, Harrogate, 24 (May/June 1992).

5. Wakeman R.J. and Sabri M.N., Scale-up procedures and test methods in solid/liquid separation: Test methods and effects of the principal variables in pressure filtration, IFPRI Research Report ARR 25-01, Separation Processes Centre Report No. 7/92, 24 (Nov. 1992). 
6. Wakeman R.J. and Sabri M.N., Scale-up procedures and test methods in solid/liquid separation: 1. Filtration of binary particle mixtures and flocculated suspensions, Proc. $14^{\text {th }}$ Annual Meeting of International Fine Particle Research Institute, Pasadena, 18 (June/July 1993).

7. Wakeman R.J., Scale-up procedures and test methods in solid/liquid separation: Filtration of binary particle mixtures and flocculated suspensions, IFPRI Research Report ARR 25-02, Separation Processes Centre Report No. 13/93, 24 (Nov. 1993).

8. Buscall R. and White L.R., The consolidation of concentrated suspensions, J. Chem. Soc. Faraday Trans. 1, 83, 873-891 (1987).

9. Landman K.A., Sirakoff C. and White L.R., Dewatering of flocculated suspensions by pressure filtration, Phys Fluids A, 3(6), 1495-1509 (1991).

10. Koenders M.A. and Wakeman R.J., A mathematical model for the initial stages of the compact formation process, (to be submitted).

11. Young I.M., in Solid/Liquid Separation Equipment Scale-up, $2^{\text {nd }}$ Edn., D.B. Purchas and R.J. Wakeman (Eds.), pp.446-484, Uplands Press and Filtration Specialists, London, (1986).

12. Tiller F.M. and Leu W.F., Basic data fitting in filtration, J. Chinese Institute Engineers, 11, 61-70 (1980).

13. Shirato M., Murase T., Iritani E., Tiller F.M. and Alciatore A.F., Filtration in the Chemical Process Industry, in Filtration, M.J. Matteson and C. Orr (Eds.), Marcel Dekker, New York, (1987).

14. Wakeman R.J., Vacuum dewatering and residual saturation of incompressible filter cakes, International J. Mineral Processing, 3, 193-206 (1976).

15. Wakeman R.J., An improved analysis for the forced gas deliquoring of filter cakes and porous media, J. Separation Process Technology, 3, 32-38 (1982).

16. Wakeman R.J., The performance of filtration post-treatment processes: 1 . The prediction and calculation of cake dewatering characteristics, Filtration and Separation, 16(6), 655-660 (1979).

17. Wakeman R.J. and Tarleton, E.S., Modelling, simulation and process design of the filter cycle, Filtration and Separation, 27(6), 412-419 (1990).

18. Wakeman R.J., The performance of filtration post-treatment processes: 2 . The estimation of cake washing characteristics, Filtration and Separation, 17(4), 67-72 (1980).

19. Wakeman R.J., Transport equations for filter cake washing, Chemical Engineering Research and Design, 64, 308-319 (1986).

20. Wakeman R.J. and Attwood G.A., Developments in the applications of cake washing theory, Filtration and Separation, 25, 272-275 (1988).

21. Wakeman R.J. and Attwood G.A., Simulations of dispersion phenomena in filter cake washing, Chemical Engineering Research and Design, 68, 161-171 (1990). 


\section{FIGURES AND TABLES}

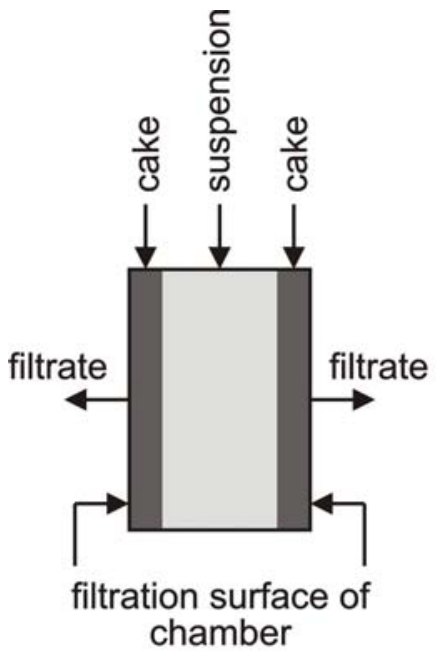

(i) Primary filtration

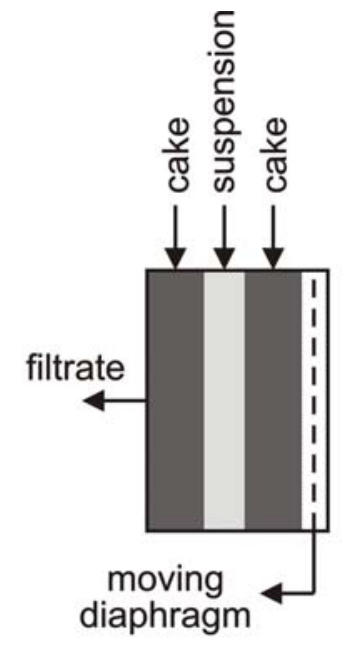

(ii) Secondary filtration

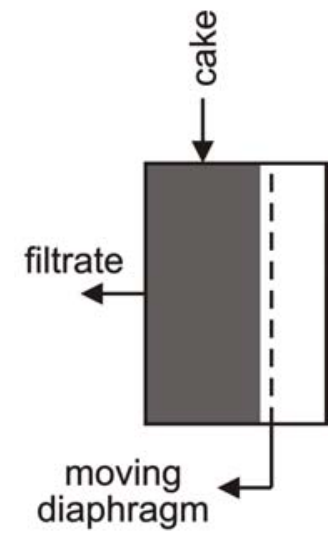

(iii) Consolidation

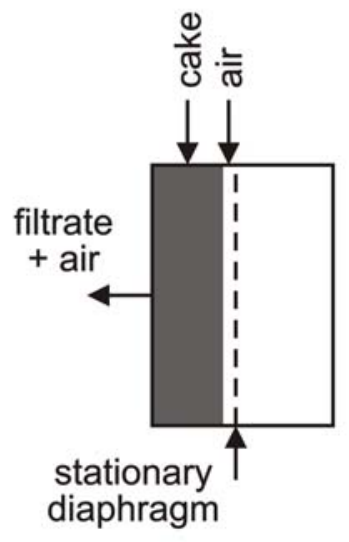

(vi) Dewatering

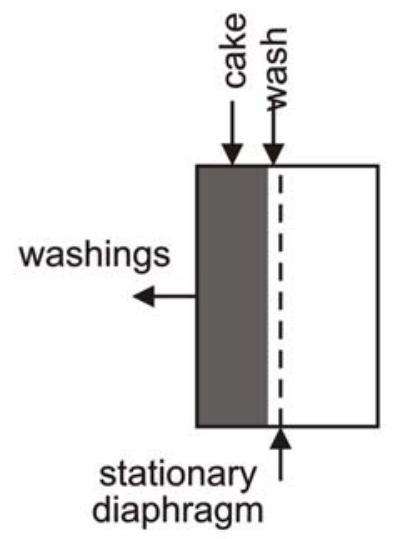

(v) Washing

Figure 1: Operations which can be carried out during a diaphragm filter press cycle. 


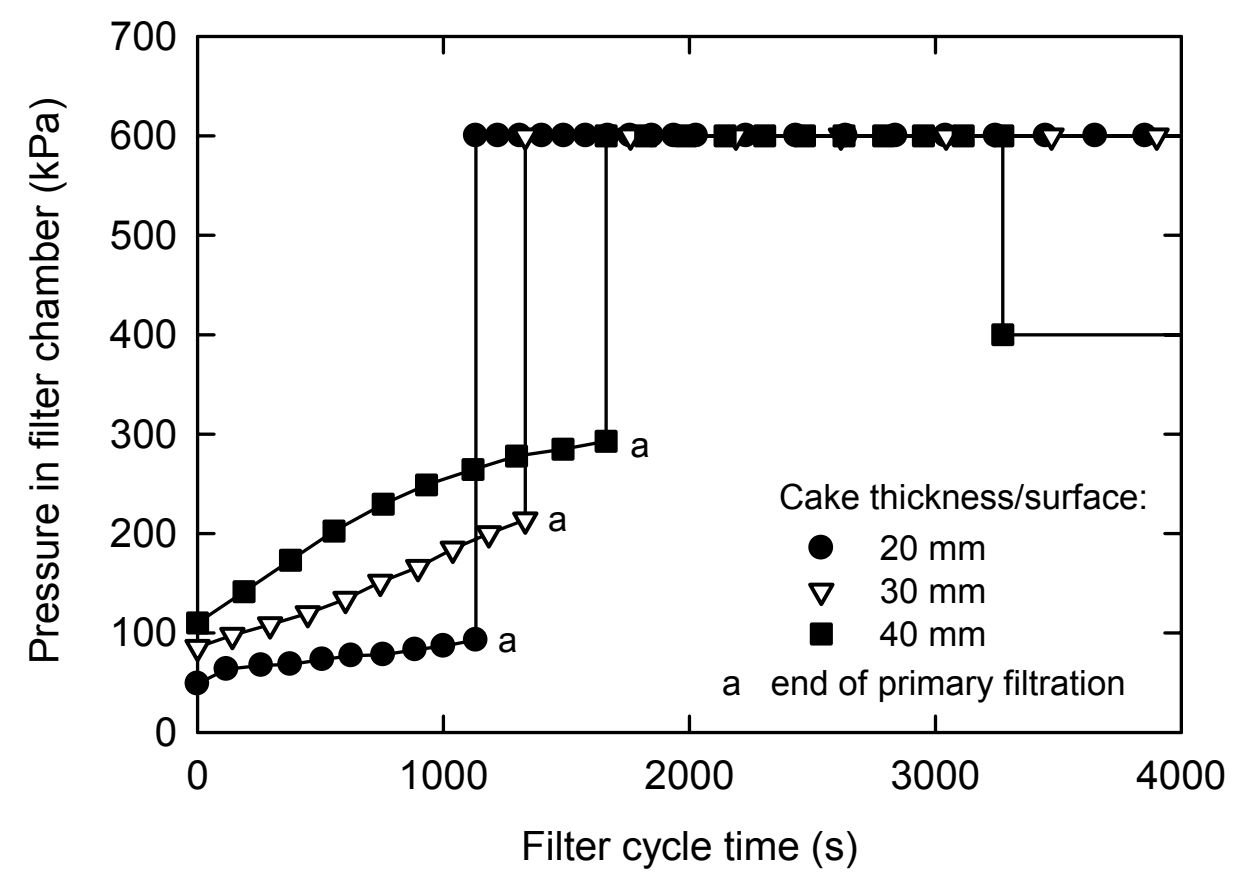

Figure 2: Variation of pressure in the filter chamber during cake formation and consolidation for different cake thicknesses formed on each filtering surface during the primary and secondary filtration stages.

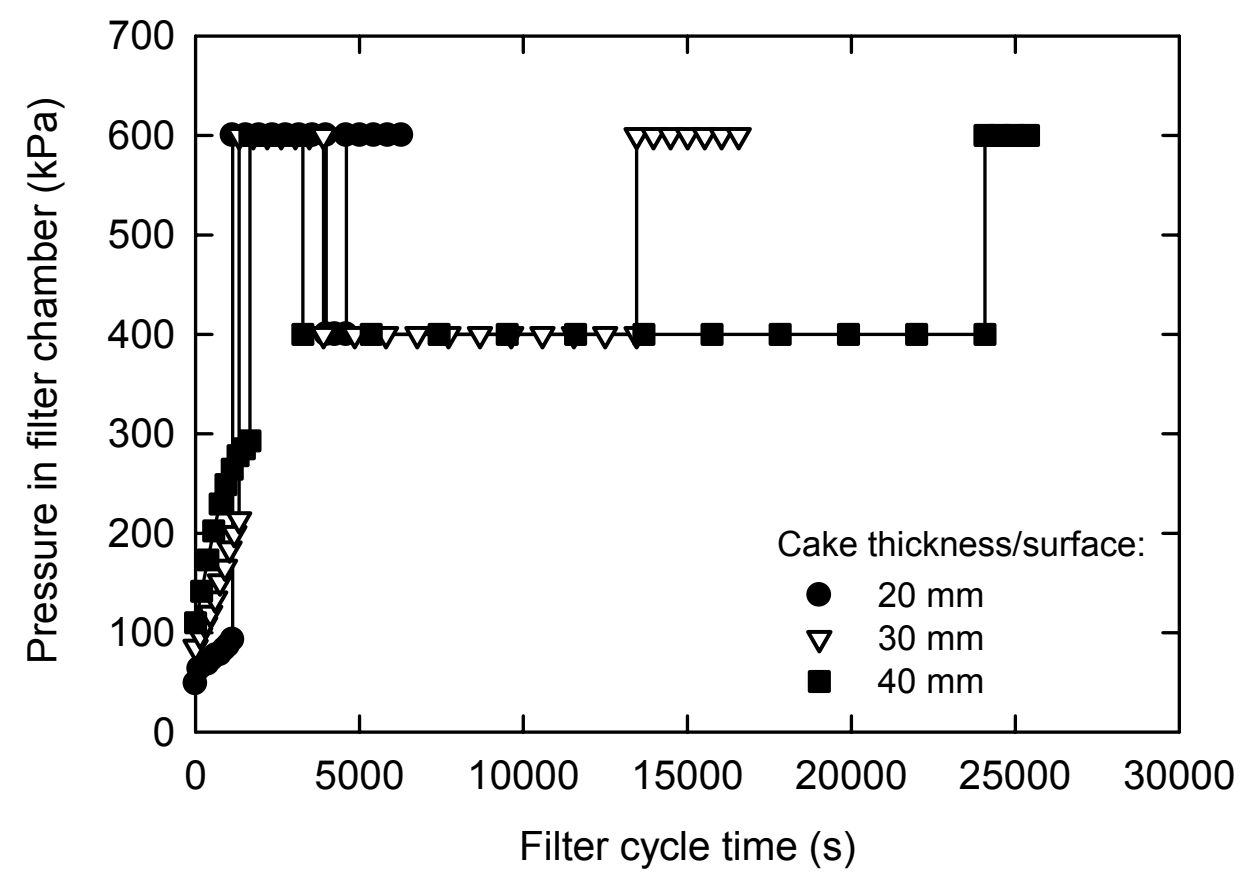

Figure 3: Variation of pressure in the filter chamber over the total operating cycle for different cake thicknesses formed on each filtering surface during the primary and secondary filtration stages. 


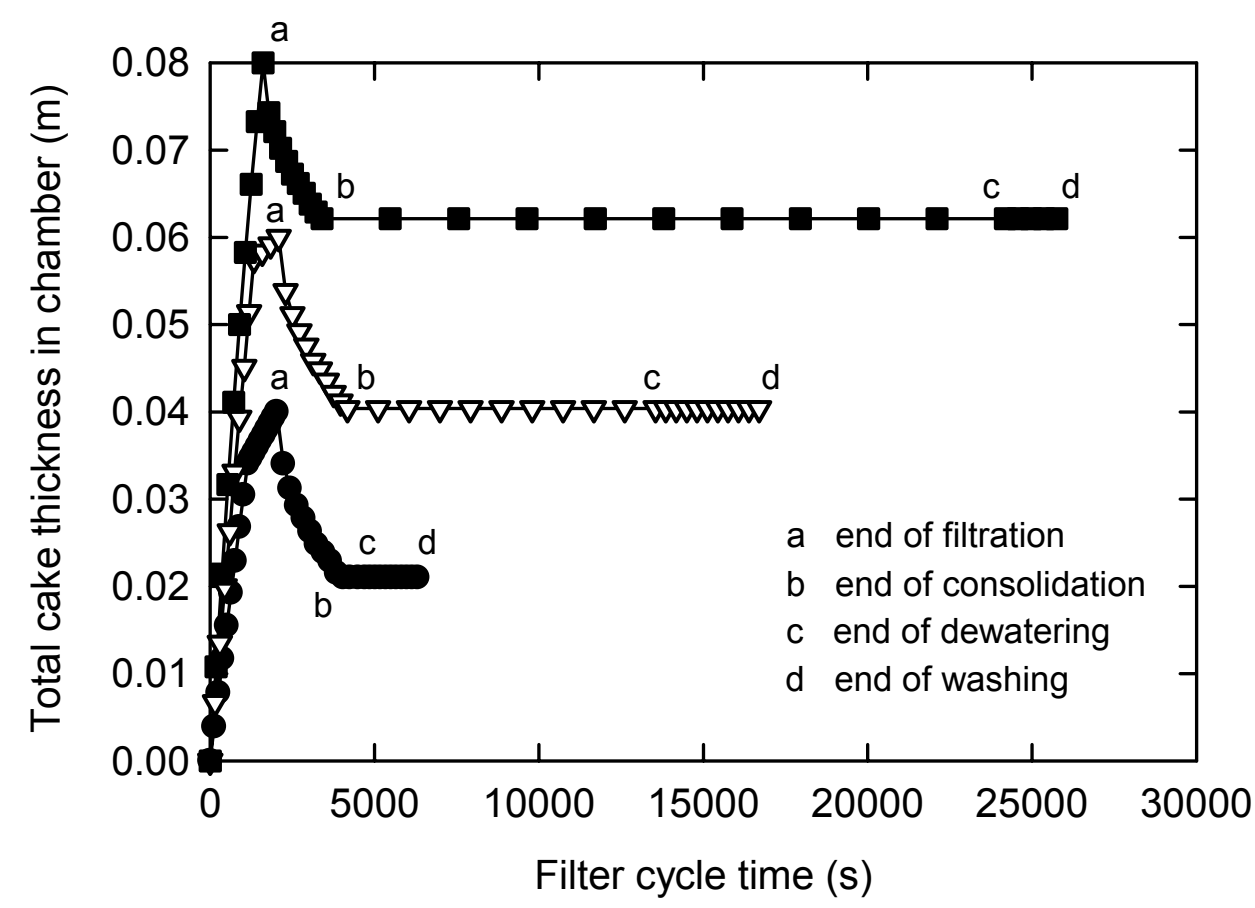

Figure 4: Variation of the total cake thickness in the filter chamber during the operating cycle of the filter.

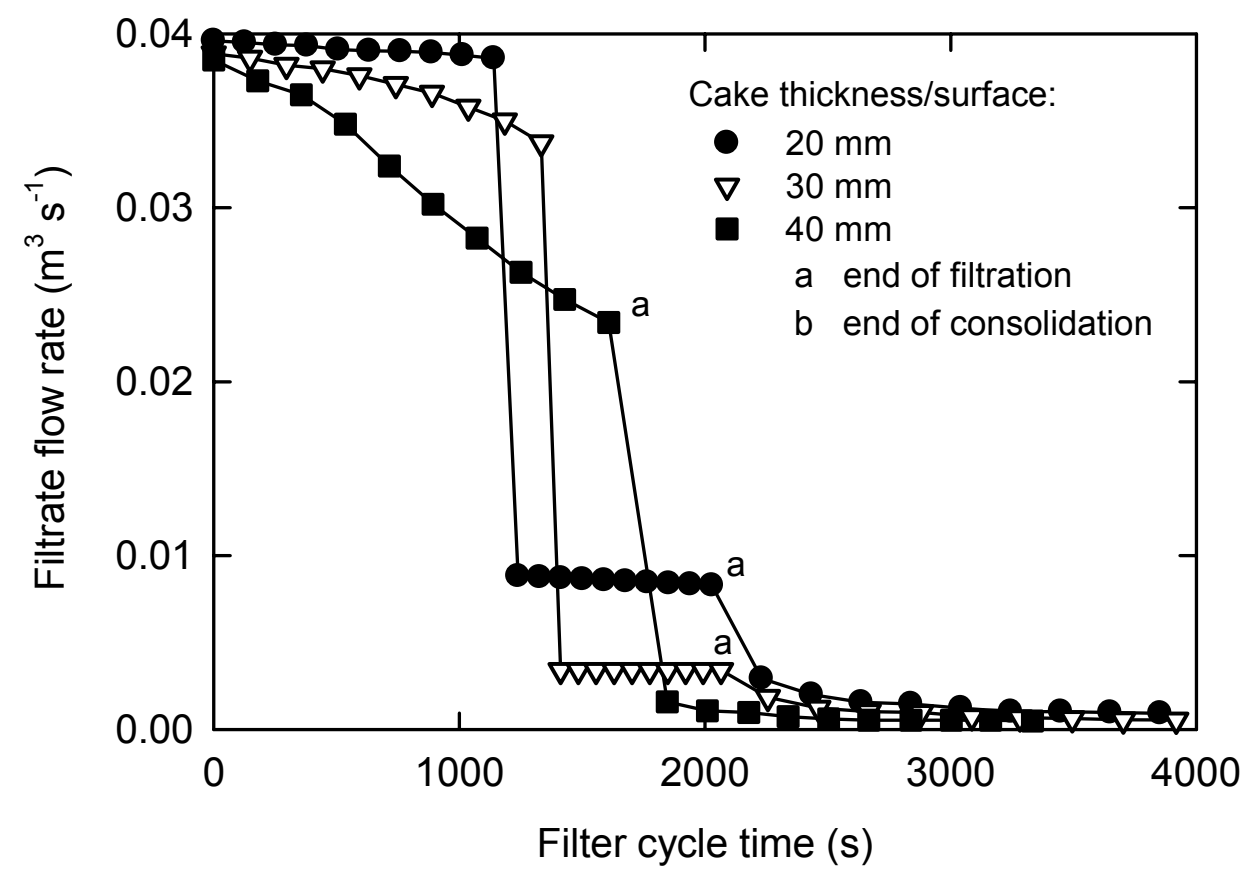

Figure 5: Decline in filtrate flow rate during the cake formation stages for different cake thicknesses formed on each filter surface during the primary and secondary filtration stages. 


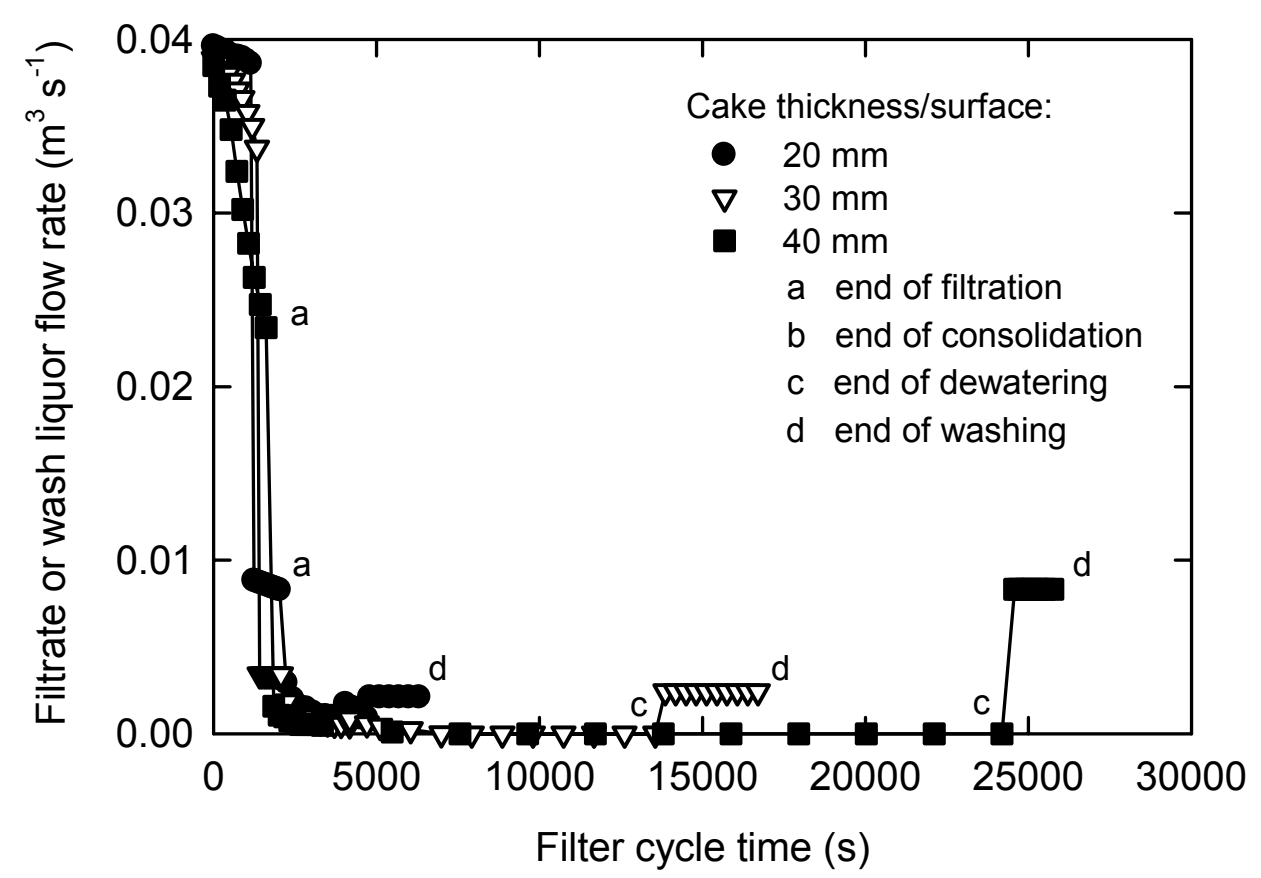

Figure 6: Variation in liquid flow rate coming from the filter press throughout the operating cycle.

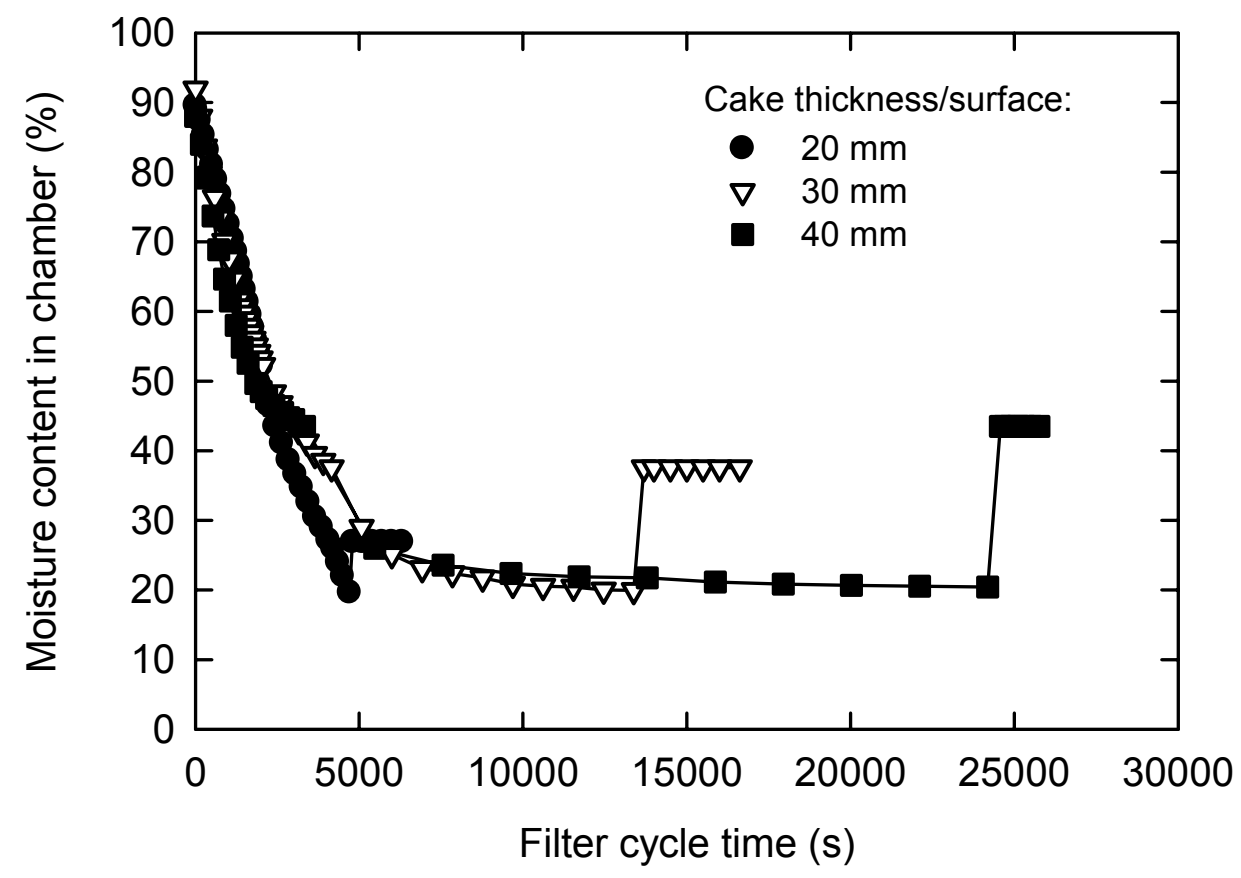

Figure 7: Variation of moisture content in the filter chamber during the operating cycle for different cake thicknesses formed on each filter surface. 


\begin{tabular}{|c|c|}
\hline Flow rate $\left(\mathrm{m}^{3} \mathrm{~s}^{-1}\right)$ & Pressure $(\mathrm{kPa})$ \\
\hline 0 & 360 \\
0.022 & 300 \\
0.03 & 250 \\
0.035 & 200 \\
0.037 & 150 \\
0.0385 & 100 \\
0.0395 & 50 \\
0.04 & 0 \\
\hline
\end{tabular}

Table 1: Pump characteristics.

\begin{tabular}{|c|c|c|c|}
\hline $\begin{array}{c}\text { Cake thickness } \\
\text { per chamber }(\mathrm{mm})\end{array}$ & $\begin{array}{c}\text { Filtration area } \\
\left(\mathrm{m}^{2}\right)\end{array}$ & $\begin{array}{c}\text { Total cycle time } \\
(\mathrm{h})\end{array}$ & $\begin{array}{c}\text { Solids production rate } \\
\left(\mathrm{kg} \mathrm{h}^{-1}\right)\end{array}$ \\
\hline 40 & 380 & 1.75 & 2857 \\
60 & 251 & 4.60 & 1087 \\
80 & 188 & 7.08 & 706 \\
\hline
\end{tabular}

Table 2: Effect of cake thickness on filter size and productivity. 